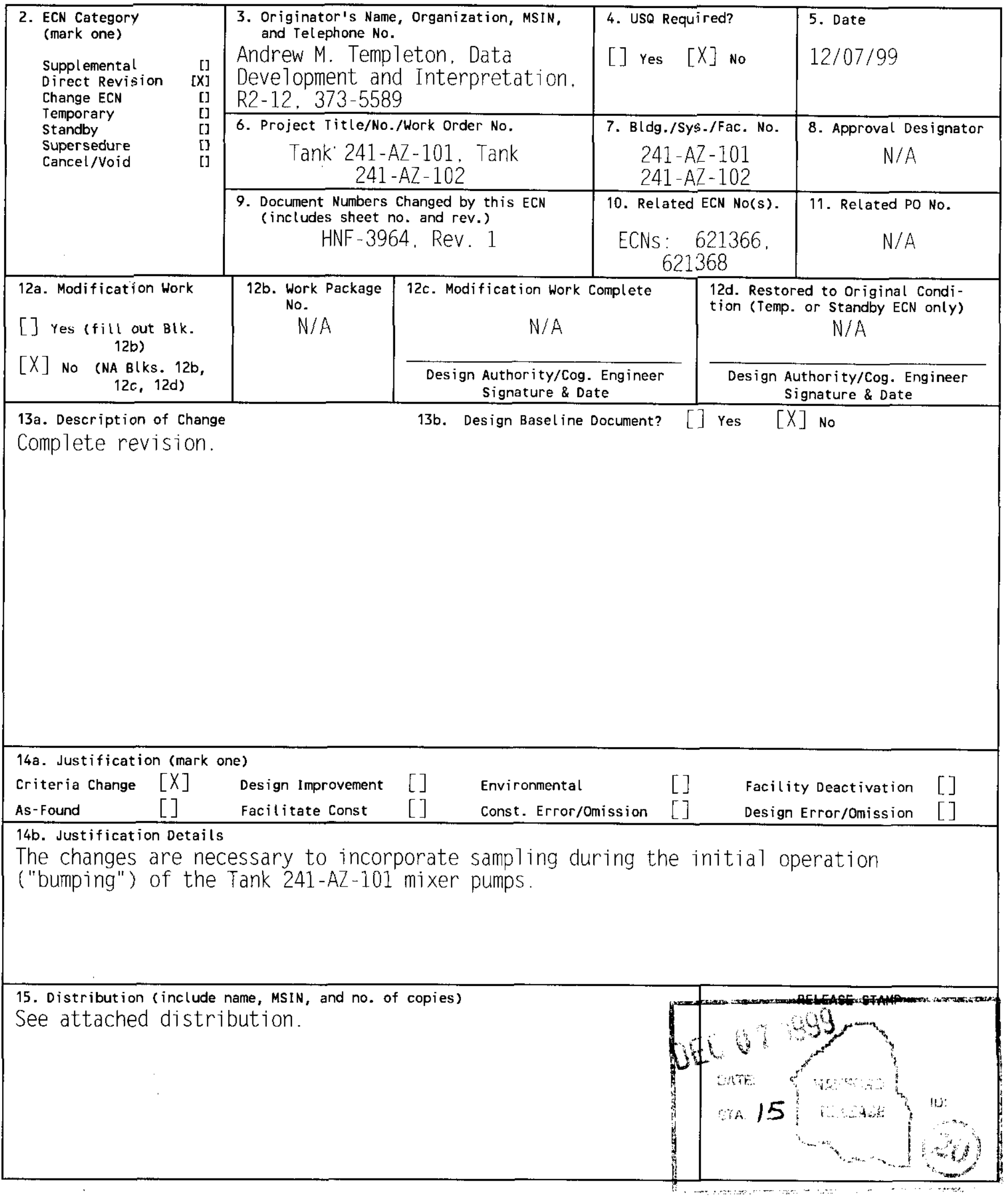




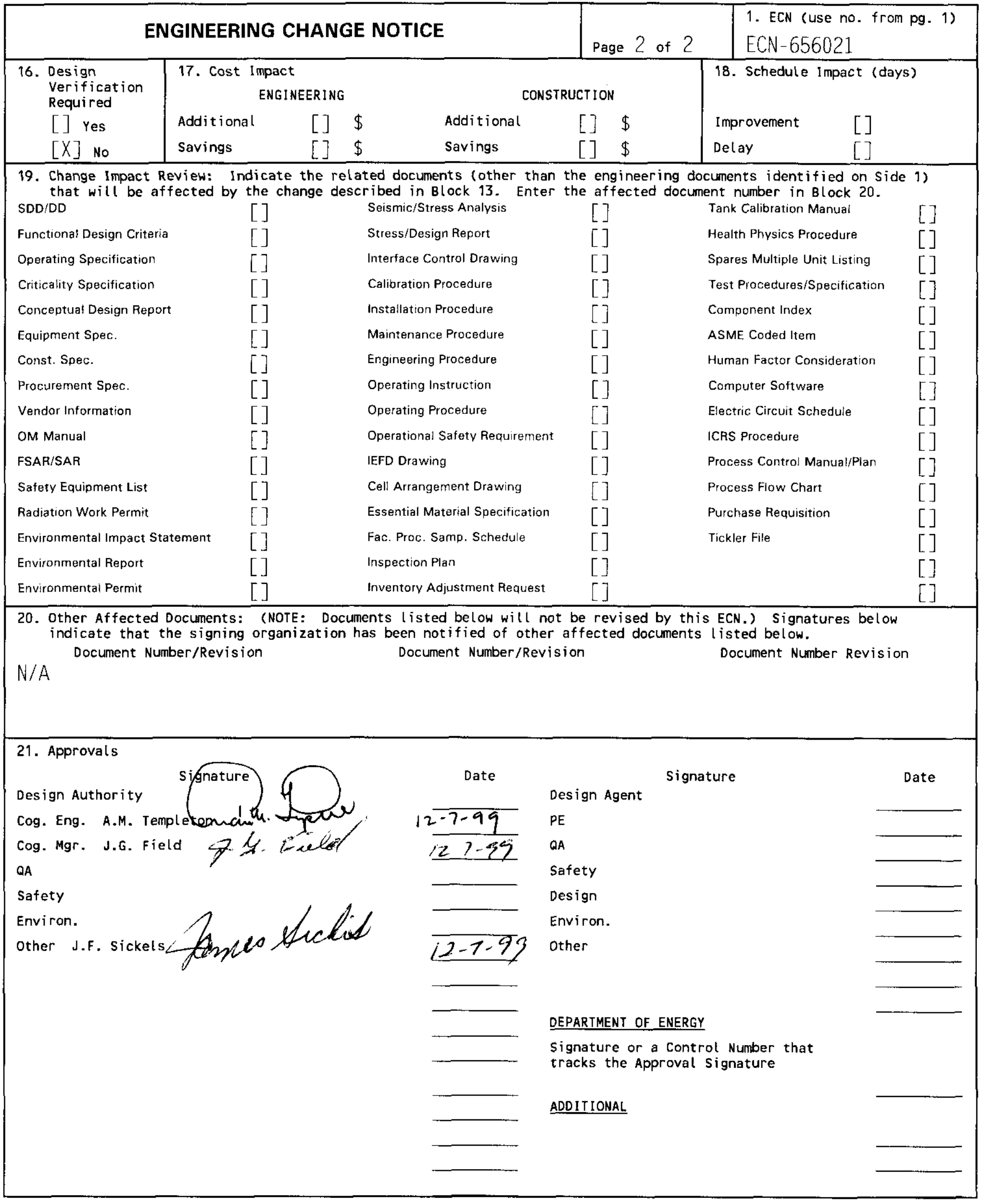




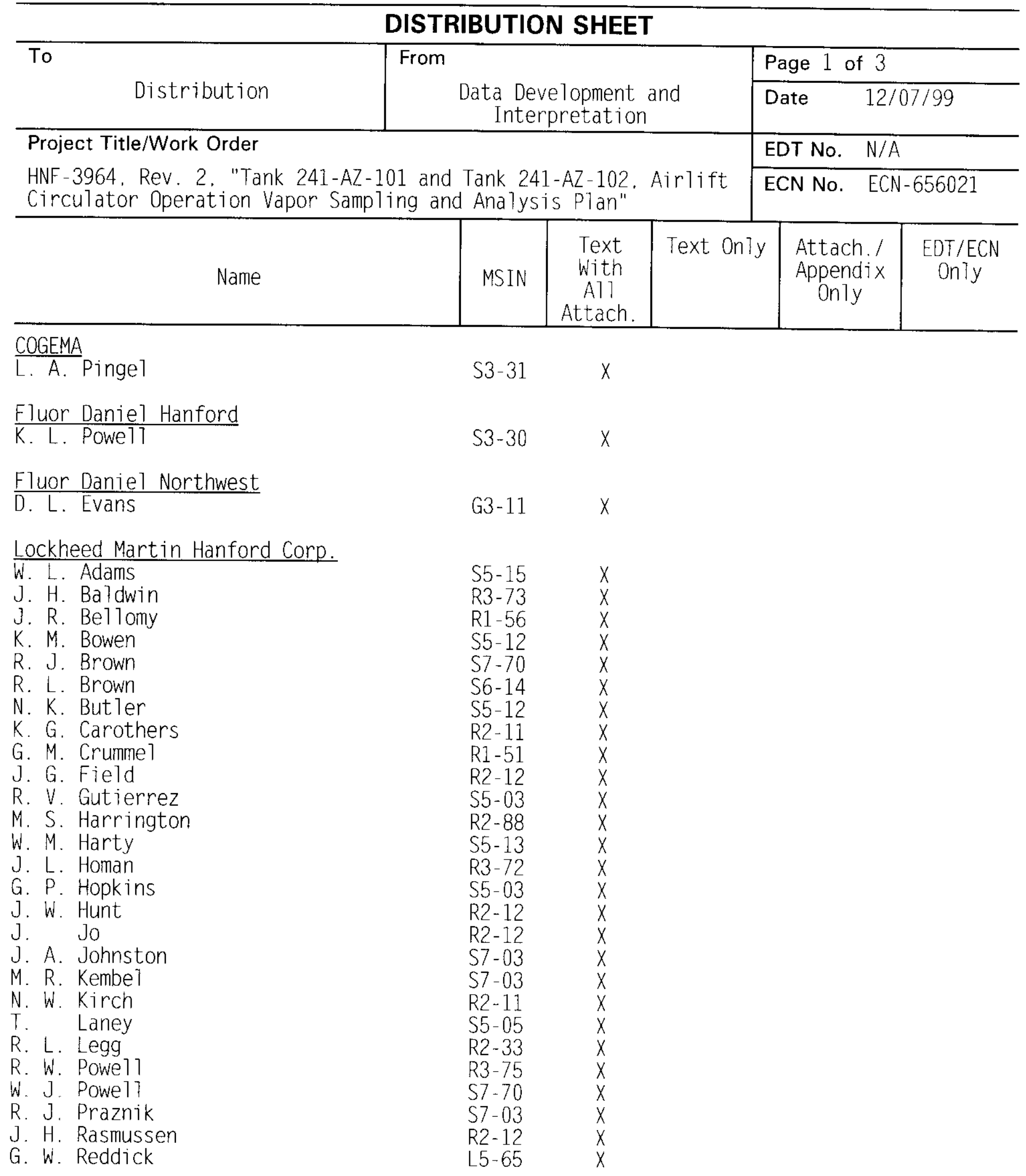


DISTRIBUTION SHEET

\begin{tabular}{|c|c|c|c|c|c|}
\hline \multirow[b]{2}{*}{ Distribution } & \multirow{2}{*}{\multicolumn{3}{|c|}{$\begin{array}{l}\text { lopment } \\
\text { retation }\end{array}$}} & \multicolumn{2}{|l|}{ Page 2 of 3} \\
\hline & & & & \multicolumn{2}{|c|}{$12 / 07 / 99$} \\
\hline \multicolumn{4}{|l|}{ Project Title/Work Order } & \multicolumn{2}{|c|}{ EDT No. N/A } \\
\hline \multicolumn{4}{|c|}{$\begin{array}{l}\text { HNF-3964, Rev. 2, "Tank 241-AZ-101 and Tank 241-AZ-102, Airlift } \\
\text { Circulator Operation Vapor Sampling and Analysis Plan" }\end{array}$} & \multicolumn{2}{|c|}{ ECN No. ECN-656021 } \\
\hline \multicolumn{2}{|l|}{ Name } & $\begin{array}{c}\text { Text } \\
\text { With } \\
\text { All } \\
\text { Attach }\end{array}$ & Text Only & $\begin{array}{l}\text { Attach./ } \\
\text { Appendix } \\
\text { Only }\end{array}$ & $\begin{array}{l}\text { EDT/ECN } \\
\text { Only }\end{array}$ \\
\hline
\end{tabular}

\section{Lockheed Martin Hanford - continued}

L. M. Sasaki

J. F. Sickels

G. A. Stanton

G. R. Tardiff

A. M. Templeton

R. R. Thompson

D. D. Wanner

T.C.S.R.C.

Lockheed Martin Services, Inc.

Central Files

Los Alamos Technical Associates

J. M. Jones

MACTEC

J. C. Guyette

R. L. Treat

R. D. Potter

Numatec Hanford Corporation

J. W. Bailey

A. B. Cartson

L. L. Lockrem

Office of River Protection

W. AbduT

DOE Reading Room

Pacific Northwest Nationa? Laboratory

J. C. Evans

J. L. Huckaby

K. L. Silvers

M. J. Truex

K. D. Wiemers
R2-12 $\quad x$

S7 $-03 \quad x$

S7-01 $\quad x$

S5-05 $\quad X$

R2 $-12 \quad x$

R2-12 $\quad X$

S7-12 $\quad X$

R1-10 $\quad X$

B1-07 $\quad X$

S5-05 $\quad x$

B1-13 $\quad X$

H6-64 $\quad X$

R3-73 $\quad x$

R3-25 $\quad x$

R3-73 $\quad x$

T6-07 $\quad X$

H6-60 $\quad X$

H2-53 $\quad X$

$k 6-96 \quad x$

K6-80 $\quad x$

K9-08 $\quad X$

K2-10 $\quad X$

H6-61 $\quad X$ 


\section{DISTRIBUTION SHEET}

\begin{tabular}{|c|c|c|c|c|c|}
\hline \multirow{2}{*}{ To $\quad$ Distribution } & \multirow{2}{*}{\multicolumn{3}{|c|}{ From }} & \multicolumn{2}{|l|}{ Page 3 of 3} \\
\hline & & & & \multicolumn{2}{|l|}{ Date } \\
\hline \multicolumn{4}{|l|}{ Project Title/Work Order } & \multicolumn{2}{|c|}{ EDT No. $\quad N / A$} \\
\hline \multicolumn{4}{|c|}{$\begin{array}{l}\text { HNF-3964, Rev. 2. "Tank } 241-A Z-101 \text { and Tank 241-AZ-102, Airlift } \\
\text { Circulator Operation Vapor Sampling and Analysis Plan" }\end{array}$} & \multicolumn{2}{|c|}{ ECN No. ECN-656021 } \\
\hline Name & MSIN & $\begin{array}{c}\text { Text } \\
\text { with } \\
\text { All } \\
\text { Attach }\end{array}$ & Text $0 n]_{\jmath}$ & $\begin{array}{l}\text { Attach./ } \\
\text { Appendix } \\
\text { Only }\end{array}$ & $\begin{array}{l}\text { EDT/ECN } \\
\text { Only }\end{array}$ \\
\hline
\end{tabular}

Waste Management Federal Services of Hanford, Inc.

C. M. Seidel

R. S. Viswanath

$\begin{array}{ll}G 1-32 & X \\ S 3-30 & X\end{array}$




\section{Tank 241-AZ-101 and Tank 241-AZ-102, Airlift Circulator Operation Vapor Sampling and Analysis Plan}

A. M. Templeton

Lockheed Martin Hanford, Corp., Richland. WA 99352

U.S. Department of Energy Contract DE-AC06-96RL13200

$\begin{array}{lll}\text { EDT/ECN: } & \text { ECN }-656021 & \text { UC: } 2070 \\ \text { Org Code: } & 74 B 20 & \text { CACN/COA: 111269/ES13 ya HMLMO } 311 \\ \text { B\&R Code: } & \text { EW } 3120074 & \text { Total Pages: } 29\end{array}$

Key Words: Tank 241-AZ-101. Tank AZ-101, AZ-101. Tank 241-AZ-102, Tank AZ-102, AZ-102. AZ Farm. Airlift Circular Operation, Vapor. Sampling, Analysis, Plan, Tank Structure

Abstract: N/A

TRADEMARK DISCLAIMER. Reference herein to any specific comercial product, process, or service by trade name, trademark, manufacturer, or otherwise, does not necessarily constitute or imply its endorsement, recommendation, or favoring by the United States Government or any agency thereof or its contractors or subcontractors.

Printed in the United States of America. To obtain copies of this document, contact: Document Control Services, P.O. Box 950, Mailstop H6-08, Richland WA 99352, Phone (509) 372-2420; Fax (509) 376-4989.

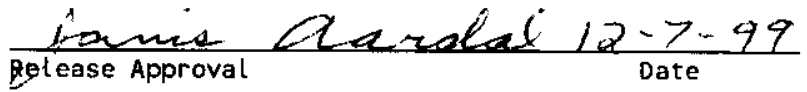

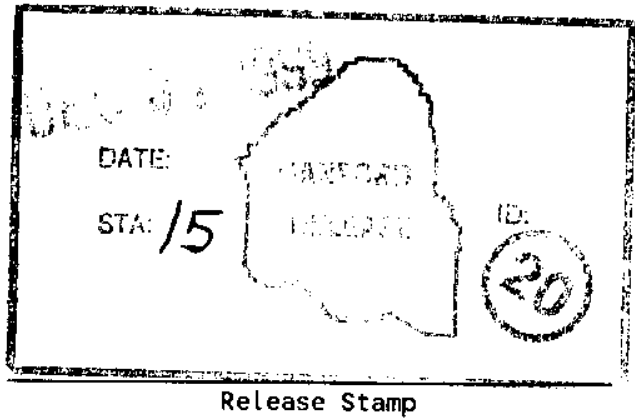

Approved for Public Release 


\section{RECORD OF REVISION}

(1) Document Number

HNF - 3964

Page 1

(2) Title

Tank 241-AZ-101 and Tank 241-AZ-102. Airlift Circulator Operation Vapor Sampling and Analysis Plan

CHANGE CONTROL RECORO

(3) Revision

0

$0-A$ RS

1 RS

2 RS
(4) Description of Change - Replace, Add, and Detete Pages

(7) Initially released $02 / 10 / 99$ on EDT611449 .

Incorporate per ECN-62.1366.

Incorporate per ECN-621368.

Incorporate per Ech.656021.
Authorized for Release

(5) Cog. Engr. (6) Cog. Mgr. Oate

L.M. Sasaki J.W. Hunt

L.M. Sasaki J.Wf Hunt

Sh Aasck: Asoof $2 / 17 / 99$

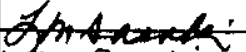

4.m. Sasaki

7 intse 3iाt

A.m. Templet on J.6. Field

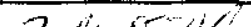


HNF-3964

Revision 2

\title{
Tank 241-AZ-101 and Tank 241-AZ-102 Airlift Circulator Operation Vapor Sampling and Analysis Plan
}

\author{
A. M. Templeton \\ Lockheed Martin Hanford Corporation
}

Date Published

February 1999

Prepared for the U.S. Department of Energy

Assistant Secretary for Environmental Management

Fluor Daniel Hanford, Inc.

P. O. Box 1000

Richland, Washington

Hanford management and Integration Contractor for the

U.S. Department of Energy under Contract DE-AC06-96-RL/3200

Approved for Public Release; Further Dissemination Unlimited 


\section{TABLE OF CONTENTS}

1.0 SAMPLING AND ANALYSIS OBJECTIVES $\ldots \ldots \ldots \ldots \ldots \ldots$

2.0 SAMPLING EVENT REQUIREMENTS $\ldots \ldots \ldots \ldots \ldots \ldots \ldots \ldots \ldots$

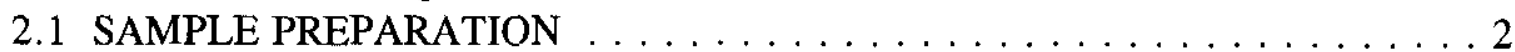

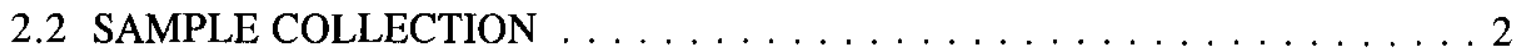

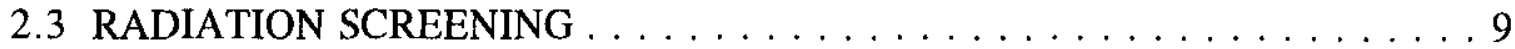

3.0 LABORATORY ANALYSIS REQUIREMENTS $\ldots \ldots \ldots \ldots \ldots \ldots \ldots \ldots$

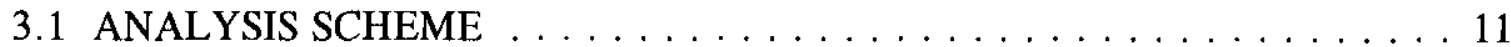

3.2 INSUFFICIENT SAMPLE RECOVERY $\ldots \ldots \ldots \ldots \ldots \ldots \ldots \ldots$

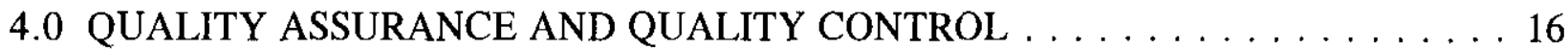

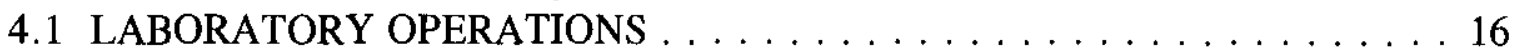

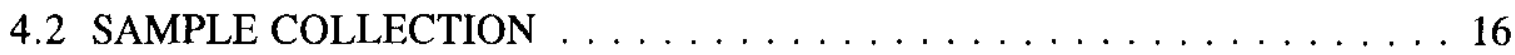

4.3 SAMPLE CUSTODY $\ldots \ldots \ldots \ldots \ldots \ldots \ldots \ldots \ldots \ldots \ldots \ldots$

5.0 EXCEPTIONS, CLARIFICATIONS, AND ASSUMPTIONS $\ldots \ldots \ldots \ldots$

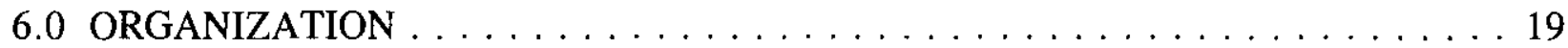

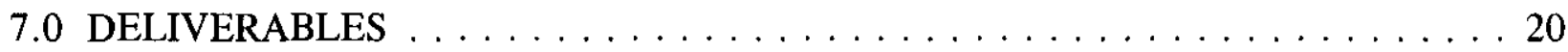

7.1 FORMAT I REPORTING $\ldots \ldots \ldots \ldots \ldots \ldots \ldots \ldots \ldots \ldots \ldots$

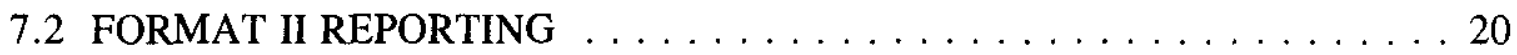

7.3 FORMAT VI REPORTING $\ldots \ldots \ldots \ldots \ldots \ldots \ldots \ldots \ldots \ldots$

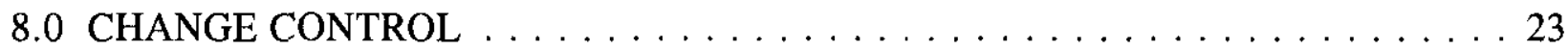

9.0 REFERENCES . . . . . . . . . . . . . . . . . . . . . . . . . 24

\section{LIST OF TABLES}

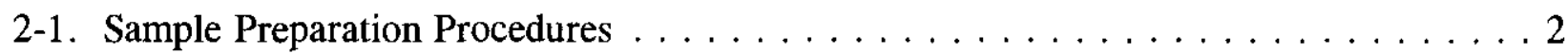

2-2. List of Samples and Activities for Tank 241-AZ-101 Headspace Sampling . . . . . . . 4

2-3. List of Samples and Activities for Tank 241-AZ-102 Headspace Sampling . . . . . . . 7

2-4. Vapor Sampling Summary . . . . . . . . . . . . . . . . . 9

3-1. Chemical and Radiological Analytical Requirements . . . . . . . . . . 12

3-2. Required Analytes: Regulatory . . . . . . . . . . . . . . . . . . . . 13

3-3. Required Analytes: Vapor Program Target Analytes . . . . . . . . . . . . . . . 14

6-1. Project Key Personnel . . . . . . . . . . . . . . . . . . . . . . . . . . . . . . 19

7-1. Data Package Required Elements . . . . . . . . . . . . . . . 21 
HNF-3964, Rev. 2

\section{LIST OF ABBREVIATIONS}

$\begin{array}{ll}\text { ALC } & \text { airlift circulator } \\ \text { CAS } & \text { Chemical Abstract Service } \\ \text { CPO } & \text { Characterization Project Operations } \\ \text { GC/MS } & \text { gas chromatography/mass spectrometry } \\ \text { HASQARD } & \text { Hanford Analytical Services Quality Assurance Requirements Document } \\ \text { IDLH } & \text { immediately dangerous to life and health } \\ \text { LFL } & \text { lower flammability limit } \\ \text { LMHC } & \text { Lockheed Martin Hanford Corporation } \\ \text { N/A } & \text { not applicable } \\ \text { NHC } & \text { Numatec Hanford Corporation } \\ \text { OTP } & \text { operational test procedure } \\ \text { ppb } & \text { parts per billion } \\ \text { ppmv } & \text { parts per million by volume } \\ \text { PHMC } & \text { Project Hanford Management Contract } \\ \text { PNNL } & \text { Pacific Northwest National Laboratory } \\ \text { QA } & \text { quality assurance } \\ \text { QC } & \text { quality control } \\ \text { SAP } & \text { sampling and analysis plan } \\ \text { SAS } & \text { Special Analytical Support } \\ \text { SHMS standard hydrogen monitoring system } \\ \text { TOC } & \text { total organic carbon } \\ \text { TWRS } & \text { Tank Waste Remediation System } \\ \text { WMH } & \text { Waste Management Hanford } \\ \text { WSCF } & \text { Waste Sampling and Characterization Facility }\end{array}$




\section{HNF-3964, Rev. 2}

\subsection{SAMPLING AND ANALYSIS OBJECTIVES}

This sampling and analysis plan (SAP) identifies characterization objectives pertaining to sample collection, laboratory analytical evaluation, and reporting requirements for vapor samples obtained during the operation of the tank 241-AZ-101 and 241-AZ-102 airlift circulators (ALCs) and during the initial operation ("bump") of the tank 241-AZ-101 mixer pumps. The purpose of the ALC operation is to support portions of the operational test procedure (OTP) for Project W-030 (OTP-W030-001) and to perform functional test in support of Project W-151. Project W-030 is the 241-A-702 ventilation upgrade project (241-AZ-702) and Project W-151 is the 241-AZ-101 Mixer Pump Test. The functional tests will check the operability of the tank 241-AZ-101 ALCs. Process Memo's \#2E98-082 and \#2E99-001 (LMHC 1999a, LMHC 1999b) direct the operation of the ALCs and the Industrial Hygiene monitoring respectively.

A series of tests will be conducted in which the ALCs in tanks 241-AZ-101 and 241-AZ-102 will be operated at different air flow rates. Vapor samples will be obtained to determine constituents that may be present in the tank headspace during ALC operation at tanks 241-AZ-101 and 241-AZ-102 as the waste is disturbed. During the testing, vapor samples will be obtained from the headspace of tanks 241-AZ-101 and 241-AZ-102 via the unused port on the standard hydrogen monitoring system (SHMS). In addition the last two vapor samples will be collected from the headspace of tank 241-AZ-101 during the operation of the mixer pumps. Each mixer pump will be operated for approximately 5 minutes. Results will be used to provide the waste feed delivery program with environmental air permitting data for tank waste disturbing activities. Because of radiological concerns, the samples will be filtered for particulates. It is recognized that this may remove some organic compounds.

The following sections provide the general methodology and procedures to be used in the preparation, retrieval, transport, analysis, and reporting of results from vapor samples retrieved during the ALC testing. 


\subsection{SAMPLING EVENT REQUIREMENTS}

The ALCs will be operated during a series of functional and operational tests. Samples will be taken prior to ALC operation and during ALC operation at each tank. ALC testing will initially be performed in tank 241-AZ-101. At tank 241-AZ-101 samples will be collected during the first two days of the ALC operation. Samples will be collected during the first three days of ALC operation at 241-AZ-102. In addition the last two samples for for tank 241-AZ101 will be collected during the initial operation ("bump") of the mixer pumps.

\subsection{SAMPLE PREPARATION}

SUMMA $^{\mathrm{Tm} 1}$ canister samples will be collected and sent to Special Analytical Support (SAS) laboratories, field and equipment blanks will accompany the samples. Tritium trap and particulate filter samples will be collected for radiation screening of the samples and will be analyzed by Waste Management Hanford (WMH) at the Waste Sampling and Characterization Facility (WSCF). Tritium traps will be supplied by SAS while the particulate filters/housing will be supplied by Characterization Project Operations (CPO). SAS will provide evacuated SUMMA canisters for collection of tank vapor samples. SAS will also provide evacuated SUMMA $^{T M}$ canisters for use in collection of particulate filters, tritium traps. Sample preparation procedures are listed in Table 2-1.

Table 2-1. Sample Preparation Procedures

\begin{tabular}{|l|l|l|}
\hline Sample container & Laboratory/Org. & Preparation Procedure \\
\hline SUMMA $^{\mathrm{TM}}$ canister & SAS & LO-080-406 \\
\hline Tritium trap & SAS & N/A \\
\hline Particulate filter & CPO & N/A \\
\hline
\end{tabular}

\subsection{SAMPLE COLLECTION}

CPO will be responsible for the collection of all SUMMA ${ }^{\mathrm{mm}}$ canister, tritium trap, and particulate filter samples from the headspace of tanks 241-AZ-101 and 241-AZ-102.

Tables 2-2, and 2-3 provide the sequence of sampling activities of the 241-AZ-101 and 241-AZ-102 headspace, respectively. In summary, the samples will be collected in the headspace of tank 241-AZ-101 prior to ALC operation, and when a flow rate of 3, 5, and $7 \mathrm{cfm}$ have been reached or at the direction of Process Engineering. In addition the last two 241-AZ-101 headspace samples will be collected during the initial start of the mixer pumps. The two pumps will each be independently operated for approximately five minutes each.

'SUMMA ${ }^{\mathrm{TM}}$ is a trademark of Moletrics, Inc., Cleveland, Ohio. 


\section{HNF-3964, Rev. 2}

Samples will be collected during each pump operation at the direction of Process Engineering. Samples will also be collected from the headspace in tank 241-AZ-102 prior to ALC operation, and when a flow rate of 3,5 , and $7 \mathrm{cfm}$ have been reached or at the direction of Process Engineering. These flow rates of 3, 5, and $7 \mathrm{cfm}$ are for each ALC. A representative from Process Engineering will be on-site during the sampling.

For each sample, the total organic carbon (TOC) concentration at the start of sample collection, the time at the start of sample collection, and the time at the end of sample collection shall be recorded. Only stainless steel, Teflon or tubing approved by Process Engineering will be used for sampling.

Each filtered tritium sample will be collected from the tank headspace prior to vapor sampling, utilizing a SUMMA canister dedicated for tritium collection. The actual vapor samples will utilize the same particulate filter used during tritium collection. The individual sample numbers, sample volumes and sample durations are listed in Tables 2-2 and 2-3 except for the particulate filter volume which will be 12 liters. The samples are summarized in Table $2-4$.

CPO sampling activities will be performed in accordance with work package ES-99-00029 for sampling of the 241-AZ-101 headspace during ALC operation, ES-99-00403 for sampling of the 241-AZ-101 headspace during mixer pump operation and work package ES-99-00039 for sampling of the tank 241-AZ-102 headspace.

The samples shall include the following quality control (QC) samples: one SUMMA ${ }^{\mathrm{TM}}$ canister ambient air field blank and one SUMMA ${ }^{\mathrm{TM}}$ canister equipment blank. The blanks are to accompany the vapor samples to the laboratory. For specific information concerning sample and blank handling, sample custody, and sample transportation, refer to the requirements in Section 4.2, 4.3 and 5.0.

Samples shall be stored by CPO until radiation screening is completed and the samples can be released for analysis. Upon completion of the radiation screening and release of the samples, SAS shall retrieve its samples from CPO. In the event the samples are determined to be radiologically contaminated by particulate material the samples will be sent to Pacific Northwest National Laboratory (PNNL).

Any decisions, observations, or deviations affecting this SAP shall be documented in controlled notebooks/or work packages and justified in the deliverable report. 
HNF-3964, Rev. 2

Table 2-2. List of Samples and Activities for Tank 241-AZ-101 Headspace Sampling (3 Sheets)

\begin{tabular}{|c|c|c|c|c|c|}
\hline $\begin{array}{l}\text { Sample } \\
\text { Code }\end{array}$ & Day & $\begin{array}{c}\text { Sample/Activity } \\
\text { Description }\end{array}$ & $\begin{array}{l}\text { Sampler Position } \\
\text { During Collection }\end{array}$ & $\begin{array}{l}\text { Sample } \\
\text { Volume }\end{array}$ & $\begin{array}{l}\text { Sample } \\
\text { Duration } \\
\text { or Rate }\end{array}$ \\
\hline 001 & Day 1 & $\begin{array}{l}\text { Collect SUMMA } \\
\text { field blank }\end{array}$ & AZ Tank Farm & $6 \mathrm{~L}$ & $2 \mathrm{~min}$. \\
\hline 002 & Day 1 & $\begin{array}{l}\text { Collect SUMMA } \\
\text { equipment blank }\end{array}$ & AZ Tank Farm & $6 \mathrm{~L}$ & $2 \mathrm{~min}$. \\
\hline 003 & Day 1 & $\begin{array}{l}\text { Collect Particulate } \\
\text { filter inline during } \\
\text { collection of Tritium } \\
\text { trap }\end{array}$ & $\begin{array}{l}\text { Tank 24I-AZ-101 } \\
\text { Headspace }\end{array}$ & --- & --- \\
\hline 004 & Day 1 & Collect Tritium trap & $\begin{array}{c}\text { Tank 241-AZ-101 } \\
\text { Headspace }\end{array}$ & $6 \mathrm{~L}$ & $2 \mathrm{~min}$. \\
\hline 005 & Day 1 & $\begin{array}{l}\text { Collect baseline } \\
\text { SUMMA } \text { TM }^{\mathrm{TM}} \text { with } \\
\text { Particulate filter } \\
\text { prior to start of ALC } \\
\text { operation }\end{array}$ & $\begin{array}{c}\text { Tank 241-AZ-101 } \\
\text { Headspace }\end{array}$ & $6 \mathrm{~L}$ & $2 \mathrm{~min}$ \\
\hline 006 & Day 1 & $\begin{array}{l}\text { Collect Particulate } \\
\text { filter inline during } \\
\text { collection of Tritium } \\
\text { trap }\end{array}$ & $\begin{array}{c}\text { Tank 241-AZ-101 } \\
\text { Headspace }\end{array}$ & --- & --- \\
\hline 007 & Day 1 & Collect Tritium trap & $\begin{array}{c}\text { Tank 241-AZ-101 } \\
\text { Headspace }\end{array}$ & $6 \mathrm{~L}$ & $2 \mathrm{~min}$. \\
\hline 008 & Day 1 & $\begin{array}{l}\text { Collect SUMMA } \\
\text { with Particulate } \\
\text { filter } 1 \text { hour after } \\
\text { establishing ALC } \\
\text { flow rate of } 3 \mathrm{cfm} \text { or } \\
\text { at the direction of } \\
\text { Process Engineering }\end{array}$ & $\begin{array}{c}\text { Tank 241-AZ-101 } \\
\text { Headspace }\end{array}$ & $6 \mathrm{~L}$ & $2 \mathrm{~min}$. \\
\hline 009 & Day $1-2$ & $\begin{array}{l}\text { Collect Particulate } \\
\text { filter inline during } \\
\text { collection of Tritium } \\
\text { trap }\end{array}$ & $\begin{array}{c}\text { Tank 241-AZ-101 } \\
\text { Headspace }\end{array}$ & -- & --- \\
\hline 010 & Day $1-2$ & Collect Tritium trap & $\begin{array}{c}\text { Tank 241-AZ-101 } \\
\text { Headspace }\end{array}$ & $6 \mathrm{~L}$ & $2 \mathrm{~min}$. \\
\hline
\end{tabular}


HNF-3964, Rev. 2

Table 2-2. List of Samples and Activities for Tank 241-AZ-101 Headspace Sampling (3 Sheets)

\begin{tabular}{|c|c|c|c|c|c|}
\hline $\begin{array}{l}\text { Sample } \\
\text { Code }\end{array}$ & Day & $\begin{array}{l}\text { Sample/Activity } \\
\text { Description }\end{array}$ & $\begin{array}{l}\text { Sampler Position } \\
\text { During Collection }\end{array}$ & $\begin{array}{l}\text { Sample } \\
\text { Volume }\end{array}$ & $\begin{array}{l}\text { Sample } \\
\text { Duration } \\
\text { or Rate }\end{array}$ \\
\hline 011 & Day 1-2 & $\begin{array}{l}\text { Collect SUMMA } \\
\text { with Particulate } \\
\text { filter at direction of } \\
\text { Process Engineering } \\
\text { (Optional) }\end{array}$ & $\begin{array}{c}\text { Tank 241-AZ-101 } \\
\text { Headspace }\end{array}$ & $6 \mathrm{~L}$ & $2 \mathrm{~min}$ \\
\hline 012 & Day $1-2$ & $\begin{array}{l}\text { Collect Particulate } \\
\text { filter inline during } \\
\text { collection of Tritium } \\
\text { trap }\end{array}$ & $\begin{array}{c}\text { Tank 241-AZ-101 } \\
\text { Headspace }\end{array}$ & -- & --- \\
\hline 013 & Day 1-2 & Collect Tritium trap & $\begin{array}{c}\text { Tank 241-AZ-101 } \\
\text { Headspace }\end{array}$ & $6 \mathrm{~L}$ & $2 \mathrm{~min}$. \\
\hline 014 & Day $1-2$ & $\begin{array}{l}\text { Collect SUMMA TM } \\
\text { with Particulate } \\
\text { filter at direction of } \\
\text { Process Engineering } \\
\text { (Optional) }\end{array}$ & $\begin{array}{c}\text { Tank 241-AZ-101 } \\
\text { Headspace }\end{array}$ & $6 \mathrm{~L}$ & $2 \mathrm{~min}$. \\
\hline 015 & Day I-2 & $\begin{array}{l}\text { Collect Particulate } \\
\text { filter inline during } \\
\text { collection of tritium } \\
\text { trap }\end{array}$ & $\begin{array}{c}\text { Tank 241-AZ-101 } \\
\text { Headspace }\end{array}$ & -- & -- \\
\hline 016 & Day $1-2$ & Collect Tritium trap & $\begin{array}{l}\text { Tank 241-AZ-101 } \\
\text { Headspace }\end{array}$ & $6 \mathrm{~L}$ & $2 \mathrm{~min}$. \\
\hline 017 & Day $1-2$ & $\begin{array}{l}\text { Collect SUMMA }{ }^{\mathrm{TM}} \\
\text { with Particulate } \\
\text { filter } 1 \text { hour after } \\
\text { establishing ALC } \\
\text { flow rate of } 5 \mathrm{cfm} \text { or } \\
\text { at the direction of } \\
\text { Process Engineering }\end{array}$ & $\begin{array}{c}\text { Tank 241-AZ-101 } \\
\text { Headspace }\end{array}$ & $6 \mathrm{~L}$ & $2 \mathrm{~min}$. \\
\hline 018 & Day $1-2$ & $\begin{array}{l}\text { Collect Particulate } \\
\text { filter inline during } \\
\text { collection of Tritium } \\
\text { trap }\end{array}$ & $\begin{array}{c}\text { Tank } 241-A Z-101 \\
\text { Headspace } \\
\text { During mixer pump } \\
\text { operation }\end{array}$ & $\ldots$ & --- \\
\hline 019 & Day $1-2$ & Collect Tritium trap & $\begin{array}{c}\text { Tank } 241-A Z-101 \\
\text { Headspace } \\
\text { During mixer pump } \\
\text { operation }\end{array}$ & $6 \mathrm{~L}$ & $2 \mathrm{~min}$. \\
\hline
\end{tabular}


HNF-3964, Rev. 2

Table 2-2. List of Samples and Activities for Tank 241-AZ-101 Headspace Sampling (3 Sheets)

\begin{tabular}{|c|c|c|c|c|c|}
\hline $\begin{array}{l}\text { Sample } \\
\text { Code }\end{array}$ & Day & $\begin{array}{c}\text { Sample/Activity } \\
\text { Description }\end{array}$ & $\begin{array}{l}\text { Sampler Position } \\
\text { During Collection }\end{array}$ & $\begin{array}{l}\text { Sample } \\
\text { Volume }\end{array}$ & $\begin{array}{l}\text { Sample } \\
\text { Duration } \\
\text { or Rate }\end{array}$ \\
\hline 020 & Day $1-2$ & $\begin{array}{l}\text { Collect SUMMATM } \\
\text { with Particulate } \\
\text { filter at direction of } \\
\text { Process Engineering } \\
\text { (Optional) }\end{array}$ & $\begin{array}{l}\text { Tank } 241-\mathrm{AZ}-101 \\
\text { Headspace } \\
\text { During mixer pump } \\
\text { operation }\end{array}$ & $6 \mathrm{~L}$ & $2 \mathrm{~min}$ \\
\hline 021 & Day 2 & $\begin{array}{l}\text { Collect Particulate } \\
\text { filter inline during } \\
\text { collection of Tritium } \\
\text { trap }\end{array}$ & $\begin{array}{l}\text { Tank } 241-\mathrm{AZ}-101 \\
\text { Headspace } \\
\text { During mixer pump } \\
\text { operation }\end{array}$ & --- & --- \\
\hline 022 & Day 2 & Collect Tritium trap & $\begin{array}{l}\text { Tank 241-AZ-101 } \\
\text { Headspace } \\
\text { During mixer pump } \\
\text { operation }\end{array}$ & $6 \mathrm{~L}$ & $2 \mathrm{~min}$. \\
\hline 023 & Day 2 & $\begin{array}{l}\text { Collect SUMMATM } \\
\text { with Particulate } \\
\text { filter } 1 \text { hour after } \\
\text { establishing ALC } \\
\text { flow rate of } 7 \mathrm{cfm} \text { or } \\
\text { at the direction of } \\
\text { Process Engineering }\end{array}$ & $\begin{array}{c}\text { Tank 241-AZ-101 } \\
\text { Headspace } \\
\text { During mixer pump } \\
\text { operation }\end{array}$ & $6 \mathrm{~L}$ & $2 \mathrm{~min}$. \\
\hline
\end{tabular}


HNF-3964, Rev. 2

Table 2-3. List of Samples and Activities for Tank 241-AZ-102 Headspace Sampling (2 Sheets)

\begin{tabular}{|c|c|c|c|c|c|}
\hline Sample Code & Day & Sample/Activity Description & $\begin{array}{l}\text { Sampler Position } \\
\text { During Collection }\end{array}$ & $\begin{array}{l}\text { Sample } \\
\text { Volume }\end{array}$ & $\begin{array}{c}\text { Sample } \\
\text { Duration or } \\
\text { Rate }\end{array}$ \\
\hline 001 & Day 1 & $\begin{array}{l}\text { Collect Particulate filter inline } \\
\text { during collection of Tritium trap }\end{array}$ & $\begin{array}{c}\text { Tank 241-AZ-102 } \\
\text { Headspace }\end{array}$ & -- & -- \\
\hline 002 & Day 1 & Collect Tritium trap & $\begin{array}{c}\text { Tank 241-AZ-102 } \\
\text { Headspace }\end{array}$ & $6 \mathrm{~L}$ & $2 \mathrm{~min}$. \\
\hline 003 & Day 1 & $\begin{array}{l}\text { Collect baseline SUMMA }{ }^{\mathrm{TM}} \text { with } \\
\text { Particulate filter prior to start of } \\
\text { ALC operation }\end{array}$ & $\begin{array}{c}\text { Tank 241-AZ-102 } \\
\text { Headspace }\end{array}$ & $6 \mathrm{~L}$ & $2 \mathrm{~min}$. \\
\hline 004 & Day 1 & $\begin{array}{l}\text { Collect Particulate filter inline } \\
\text { during collection of Tritium trap }\end{array}$ & $\begin{array}{c}\text { Tank 241-AZ-102 } \\
\text { Headspace }\end{array}$ & -- & -- \\
\hline 005 & Day 1 & Collect Tritium trap & $\begin{array}{c}\text { Tank 241-AZ-102 } \\
\text { Headspace }\end{array}$ & $6 \mathrm{~L}$ & $2 \mathrm{~min}$. \\
\hline 006 & Day 1 & $\begin{array}{l}\text { Collect SUMMA } \\
\text { filter } \\
1 \text { hour after establishing ALC flow } \\
\text { rate of } 3 \mathrm{cfm} \text { or at the direction of } \\
\text { Process Engineering }\end{array}$ & $\begin{array}{c}\text { Tank 241-AZ-102 } \\
\text { Headspace }\end{array}$ & $6 \mathrm{~L}$ & $2 \mathrm{~min}$. \\
\hline 007 & Day $1-2$ & $\begin{array}{l}\text { Collect Particulate filter inline } \\
\text { during collection of Tritium trap }\end{array}$ & $\begin{array}{c}\text { Tank 241-AZ-102 } \\
\text { Headspace }\end{array}$ & --. & -- \\
\hline 008 & Day 1-2 & Collect Tritium Trap & $\begin{array}{c}\text { Tank 241-AZ-102 } \\
\text { Headspace }\end{array}$ & $6 \mathrm{~L}$ & $2 \mathrm{~min}$. \\
\hline 009 & Day 1-2 & $\begin{array}{l}\text { Collect SUMMA }{ }^{\mathrm{TM}} \text { with Particulate } \\
\text { filter at direction of Process } \\
\text { Engineering (Optional) }\end{array}$ & $\begin{array}{c}\text { Tank } 241-A Z-102 \\
\text { Headspace }\end{array}$ & $6 \mathrm{~L}$ & $2 \mathrm{~min}$. \\
\hline 010 & Day 1-2 & $\begin{array}{l}\text { Collect Particulate filter inline } \\
\text { during collection of Tritium trap }\end{array}$ & $\begin{array}{c}\text { Tank 241-AZ-102 } \\
\text { Headspace }\end{array}$ & $\ldots$ & --- \\
\hline 011 & Day 1-2 & Collect Tritium trap & $\begin{array}{c}\text { Tank 241-AZ-102 } \\
\text { Headspace }\end{array}$ & $6 \mathrm{~L}$ & $2 \mathrm{~min}$. \\
\hline 012 & Day $1-2$ & $\begin{array}{l}\text { Collect SUMMA }{ }^{\mathrm{TM}} \text { with Particulate } \\
\text { filter } \\
1 \text { hour after establishing ALC flow } \\
\text { rate of } 5 \mathrm{cfm} \text { or at the direction of } \\
\text { Process Engineering }\end{array}$ & $\begin{array}{c}\text { Tank 241-AZ-102 } \\
\text { Headspace }\end{array}$ & $6 \mathrm{~L}$ & $2 \mathrm{~min}$ \\
\hline 013 & Day 1-2 & $\begin{array}{l}\text { Collect Particulate filter inline } \\
\text { during collection of Tritium trap }\end{array}$ & $\begin{array}{c}\text { Tank 241-AZ-102 } \\
\text { Headspace }\end{array}$ & $\cdots$ & -- \\
\hline 014 & Day $1-2$ & Collect Tritium trap & $\begin{array}{l}\text { Tank 241-AZ-102 } \\
\text { Headspace }\end{array}$ & $6 \mathrm{~L}$ & $2 \mathrm{~min}$. \\
\hline
\end{tabular}


HNF-3964, Rev. 2

Table 2-3. List of Samples and Activities for Tank 241-AZ-102 Headspace Sampling (2 Sheets)

\begin{tabular}{|c|c|c|c|c|c|}
\hline Sample Code & Day & Sample/Activity Description & $\begin{array}{l}\text { Sampler Position } \\
\text { During Collection }\end{array}$ & $\begin{array}{l}\text { Sample } \\
\text { Volume }\end{array}$ & $\begin{array}{l}\text { Sample } \\
\text { Duration or } \\
\text { Rate }\end{array}$ \\
\hline 015 & Day 1-2 & $\begin{array}{l}\text { Collect SUMMA }{ }^{\mathrm{TM}} \text { with Particulate } \\
\text { filter at the direction of Process } \\
\text { Engineering (Optional) }\end{array}$ & $\begin{array}{l}\text { Tank 241-AZ-102 } \\
\text { Headspace }\end{array}$ & $6 \mathrm{~L}$ & $2 \mathrm{~min}$. \\
\hline 016 & Day 2-3 & $\begin{array}{l}\text { Collect Particulate filter inline } \\
\text { during collection Tritium trap }\end{array}$ & $\begin{array}{c}\text { Tank 241-AZ-102 } \\
\text { Headspace }\end{array}$ & $-\cdots$ & --- \\
\hline 017 & Day $2-3$ & Collect Tritium trap & $\begin{array}{c}\text { Tank 241-AZ-102 } \\
\text { Headspace }\end{array}$ & $6 \mathrm{~L}$ & $2 \mathrm{~min}$. \\
\hline 018 & Day $2-3$ & $\begin{array}{l}\text { Collect SUMMA }{ }^{\mathrm{TM}} \text { with Particulate } \\
\text { filter } \\
1 \text { hour after establishing a ALC } \\
\text { flow rate of } 7 \mathrm{cfm} \text { or at the direction } \\
\text { of Process Engineering }\end{array}$ & $\begin{array}{c}\text { Tank } 241-A Z-102 \\
\text { Headspace }\end{array}$ & $6 \mathrm{~L}$ & $2 \mathrm{~min}$. \\
\hline 019 & Day $2-3$ & $\begin{array}{l}\text { Collect Particulate filter inline } \\
\text { during collection of Tritium trap }\end{array}$ & $\begin{array}{c}\text { Tank 241-AZ-102 } \\
\text { Headspace }\end{array}$ & -- & -- \\
\hline 020 & Day $2-3$ & Collect Tritium trap & $\begin{array}{c}\text { Tank 241-AZ-102 } \\
\text { Headspace }\end{array}$ & $6 \mathrm{~L}$ & $2 \mathrm{~min}$. \\
\hline 021 & Day 2-3 & $\begin{array}{l}\text { Collect SUMMA }{ }^{\mathrm{TM}} \text { with Particulate } \\
\text { filter at the direction of Process } \\
\text { Engineering (Optional) }\end{array}$ & $\begin{array}{c}\text { Tank } 241-A Z-102 \\
\text { Headspace }\end{array}$ & $6 \mathrm{~L}$ & $2 \mathrm{~min}$. \\
\hline
\end{tabular}


HNF-3964, Rev. 2

Table 2-4. Vapor Sampling Summary

\begin{tabular}{|c|c|c|c|c|}
\hline $\begin{array}{l}\text { Sample } \\
\text { location }\end{array}$ & Sample time & Sample type & Sample container & $\begin{array}{l}\text { Number of } \\
\text { samples. }\end{array}$ \\
\hline AZ Tank Farms & Baseline & $\begin{array}{l}\text { Field } \\
\text { blank/Ambient } \\
\text { Air }\end{array}$ & SUMMA'TM & 1 \\
\hline AZ Tank Farms & Baseline & Equipment blank & SUMMA & 1 \\
\hline \multirow{9}{*}{$\begin{array}{l}\text { Tank 241-AZ- } \\
101 \text { Headspace }\end{array}$} & \multirow[t]{3}{*}{ Baseline } & \multirow[t]{3}{*}{ Tank vapor } & SUMMA $^{\mathrm{TM}}$ & 1 \\
\hline & & & $\begin{array}{l}\text { Particulate Filter } \\
\text { Assembly }^{1}\end{array}$ & 1 \\
\hline & & & Tritium Trap & 1 \\
\hline & \multirow{3}{*}{$\begin{array}{l}\text { During } \\
\text { operation of air } \\
\text { lift circulators }\end{array}$} & \multirow[t]{3}{*}{ Tank vapor } & SUMMA $^{\mathrm{TM}}$ & 4 \\
\hline & & & $\begin{array}{l}\text { Particulate Filter } \\
\text { Assembly }{ }^{1}\end{array}$ & 4 \\
\hline & & & Tritium trap & 4 \\
\hline & \multirow{3}{*}{$\begin{array}{l}\text { During } \\
\text { operation of } \\
\text { mixer pumps }\end{array}$} & \multirow[t]{3}{*}{ Tank vapor } & SUMMA $^{\mathrm{TM}}$ & 2 \\
\hline & & & $\begin{array}{l}\text { Particulate Filter } \\
\text { Assembly }\end{array}$ & 2 \\
\hline & & & Tritium trap & 2 \\
\hline \multirow{6}{*}{$\begin{array}{l}\text { Tank } 241-A Z- \\
102 \text { Headspace }\end{array}$} & \multirow[t]{3}{*}{ Baseline } & \multirow[t]{3}{*}{ Tank vapor } & SUMMA $^{\mathrm{TM}}$ & 1 \\
\hline & & & $\begin{array}{l}\text { Particulate Filter } \\
\text { Assembly! }\end{array}$ & 1 \\
\hline & & & Tritium Trap & 1 \\
\hline & \multirow{3}{*}{$\begin{array}{l}\text { During } \\
\text { operation of air } \\
\text { lift circulators }\end{array}$} & \multirow[t]{3}{*}{ Tank vapor } & SUMMA & 3 to 6 \\
\hline & & & Tritium trap & 3 to 6 \\
\hline & & & $\begin{array}{l}\text { Particulate filter } \\
\text { Assembly }\end{array}$ & 3 to 6 \\
\hline
\end{tabular}

Notes:

baseline $=$ prior to ALC startup

'Each particulate filter assembly contains an upstream particulate filter and a downstream particulate filter. 


\subsection{RADIATION SCREENING}

Tritium trap samples will be collected from tank 241-AZ-101 and 241-AZ-102 headspace and analyzed for tritium. Particulate filter samples will be collected from the 241-AZ-101 and 241AZ-102 headspace and analyzed for total alpha, total beta, and gamma energy analyses. The results of these analyses will be used to allow the samples to obtain a radiological release and ensure that the samples meet the SAS laboratory acceptance criteria. Tritium traps will be supplied by SAS while the particulate filters/housing will be supplied by CPO. The particulate filters will contain two individual filters. The particulate samples will be collected inline during the collection of tritium traps and each individual SUMMA ${ }^{\mathrm{TM}}$ canister. The samples will be collected by connecting the filters and traps in assembled housings to the sample collection tubing upstream of the tritium trap or SUMMA ${ }^{\mathrm{TM}}$. Survey steps sufficient to confirm the absence of contamination will be included in sampling work packages.

The results from the radiation screening are submitted to and shall be evaluated by SAS and SAS shall provide a Format II report to each analytical laboratory (see Section 7.2). 
HNF-3964, Rev. 2

\subsection{LABORATORY ANALYSIS REQUIREMENTS}

The responsibilities of the analytical laboratories are given in this section. Additional quality control and deliverable requirements are given in Sections 4.0 and 7.0.

\subsection{ANALYSIS SCHEME}

Vapor samples shall be analyzed by the laboratory (SAS ) in accordance with Table 3-1. SUMMA ${ }^{\mathrm{TM}}$ canisters shall be analyzed for hydrogen, nitrous oxide, methane, carbon monoxide, carbon dioxide, and selected organic analytes.

Required analytes are those listed in Tables 3-2 and 3-3. Quantitation limit goals for the Class A and B toxic air pollutants in these tables are documented in Mulkey and Markillie (1995). In addition to the compounds listed, a determination is to be made for all other peaks that are at least $5 \mathrm{ppb}$ based on the nearest internal standard. If possible, peaks smaller than 10 percent of the nearest internal standard should also be identified. All major constituents in the sample should be identified.

At a minimum, the laboratory shall analyze all equipment blanks, field blanks, baseline samples, and the following samples obtained during the operation of the ALCs in tanks 241-AZ-101 and 241-AZ-102: (1) three sets of samples from 241-AZ-101 headspace and (2) three sets of samples from the tank 241-AZ-102 headspace.

Tritium traps and particulate filter samples shall be analyzed at the WSCF as discussed in Section 2.3 and in accordance with Table 3-2.

\subsection{INSUFFICIENT SAMPLE RECOVERY}

Contact Process Engineering for programmatic directions if there are any problems with the samples received for analysis. 
HNF-3964, Rev. 1

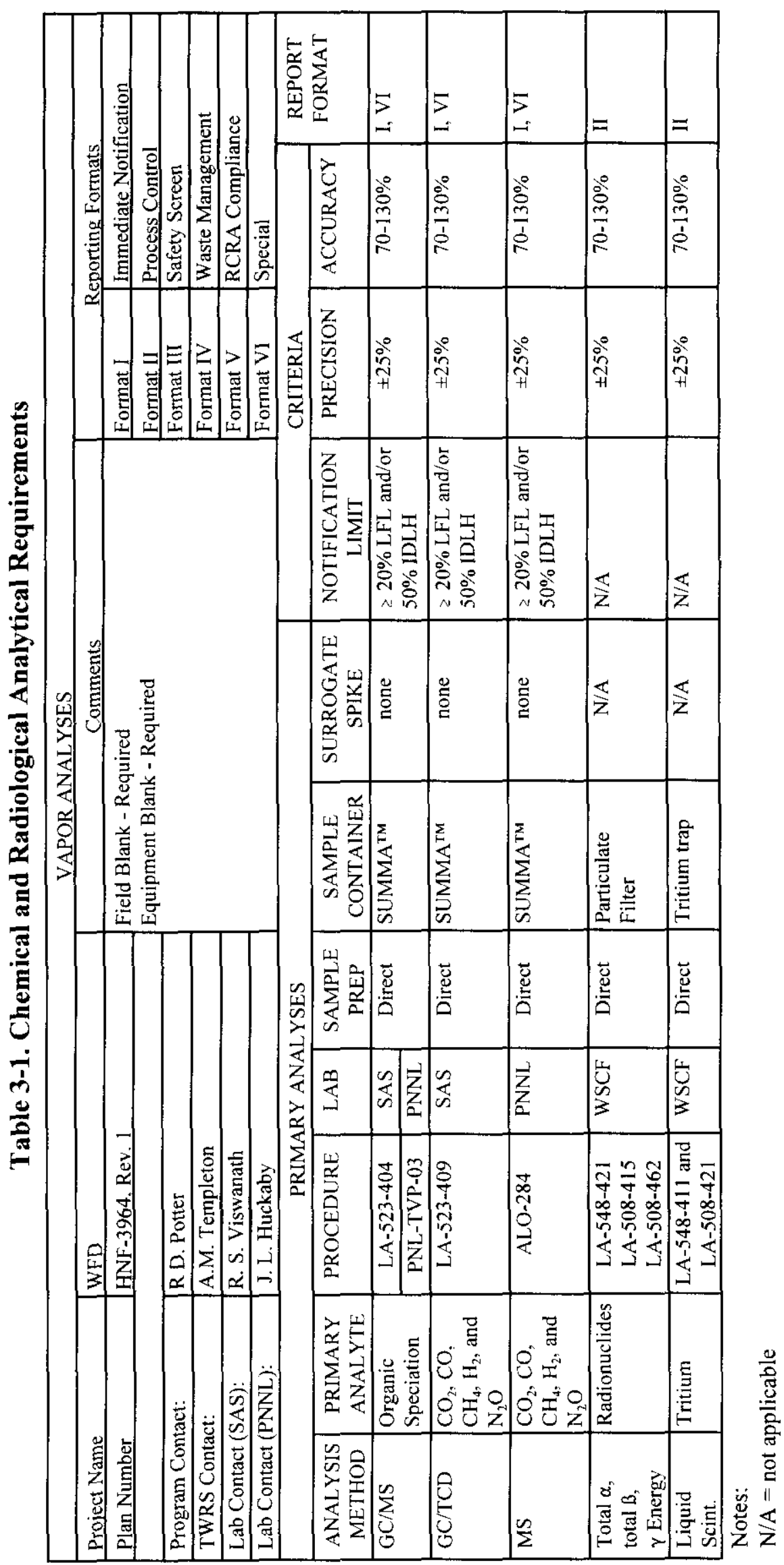


HNF-3964, Rev. 2

Table 3-2. Required Analytes: Regulatory

\begin{tabular}{|l|r|r|r|r|r|}
\hline \multicolumn{1}{|c|}{ Analyte } & \multicolumn{1}{c|}{$\begin{array}{c}\text { CAS } \\
\text { Number }\end{array}$} & $\begin{array}{r}\text { LFL } \\
\text { (ppmv) }\end{array}$ & $\begin{array}{r}\text { LFL } \\
\text { (ppmv) }\end{array}$ & $\begin{array}{r}\text { IDLH } \\
\text { (ppm) }\end{array}$ & $\begin{array}{r}\text { 50\% } \\
\text { (ppmv) }\end{array}$ \\
\hline 1,3 Butadiene & $106-99-0$ & 20,000 & 4,000 & 2,000 & 1000 \\
\hline Chloroform & $67-66-3$ & N/A & N/A & 500 & 250 \\
\hline 1,4 Dioxane & $123-91-1$ & 20,000 & 4,000 & 500 & 250 \\
\hline Benzene & $71-43-2$ & 12,000 & 2,400 & 500 & 250 \\
\hline Carbon tetrachloride & $56-23-5$ & N/A & N/A & 200 & 100 \\
\hline $\begin{array}{l}\text { Dichloromethane (Methylene } \\
\text { chloride) }\end{array}$ & $75-09-2$ & 130,000 & 26,000 & 2,300 & 1,150 \\
\hline Acetaldehyde & $75-07-1$ & 40,000 & 8,000 & 2,000 & 1,000 \\
\hline $\begin{array}{l}\text { Perchloroethylene } \\
\text { (Tetrachloroethylene) }\end{array}$ & $127-18-4$ & N/A & N/A & 150 & 75 \\
\hline Hexane & $110-54-3$ & 12,000 & 2,400 & 1,100 & 550 \\
\hline Acetone & $67-64-1$ & 25,000 & 5,000 & 2,500 & 1,250 \\
\hline n-Butanol & $71-36-3$ & 14,000 & 2,800 & 1,400 & 700 \\
\hline Nitrous oxide & $10024-97-2$ & N/A & N/A & N/A & N/A \\
\hline 3-Heptanone (Ethyl butyl ketone) & $106-35-4$ & N/A & N/A & 1,000 & 500 \\
\hline Nonane & $111-84-2$ & 8,500 & 1,700 & N/A & N/A \\
\hline 4-Heptanone (Dipropyl ketone) & $123-19-3$ & N/A & N/A & N/A & N/A \\
\hline n-Heptane & $142-82-5$ & 10,500 & 2,100 & 750 & 375 \\
\hline
\end{tabular}

Note:

$\mathrm{N} / \mathrm{A}=$ not applicable or not available 
HNF-3964, Rev. 2

Table 3-3. Required Analytes: Vapor Program Target Analytes (2 Sheets)

\begin{tabular}{|c|c|c|c|c|c|}
\hline Analyte & $\begin{array}{c}\text { CAS } \\
\text { Number }\end{array}$ & $\begin{array}{l}\text { LFL } \\
\text { (ppmv) }\end{array}$ & $\begin{array}{r}20 \% \\
\text { LFL } \\
\text { (ppmv) }\end{array}$ & $\begin{array}{l}\text { IDLH } \\
\text { (ppmv) }\end{array}$ & $\begin{array}{c}50 \% \\
\text { IDLH } \\
\text { (ppmv) }\end{array}$ \\
\hline Methane, $\mathrm{CH}_{4}$ & $74-82-8$ & 50,000 & 10,000 & N/A & N/A \\
\hline Carbon dioxide, $\mathrm{CO}_{2}$ & $124-38-9$ & N/A & $\mathrm{N} / \mathrm{A}$ & 40,000 & 20,000 \\
\hline Carbon monoxide, $\mathrm{CO}$ & $630-08-0$ & 125,000 & 25,000 & 1,200 & 600 \\
\hline Nitrous oxide, $\mathrm{N}_{2} \mathrm{O}$ & $10024-97-2$ & N/A & $\mathrm{N} / \mathrm{A}$ & N/A & N/A \\
\hline Hydrogen, $\mathrm{H}_{2}$ & 1333-74-0 & 40,000 & 8,000 & N/A & N/A \\
\hline Dichlorodifluoromethane (Freon 12) & $75-71-8$ & N/A & $\mathrm{N} / \mathrm{A}$ & 15,000 & 7,500 \\
\hline Methyl chloride & 74-87-3 & 81,000 & 16,200 & 2,000 & 1,000 \\
\hline n-Butane & $106-97-8$ & 18,000 & 3,600 & N/A & N/A \\
\hline Ethyl chloride & $75-00-3$ & 38,000 & 7,600 & 3,800 & 1,900 \\
\hline Ethanol & 64-17-5 & 33,000 & 6,600 & 3,300 & 1,650 \\
\hline Trichlorofluoromethane (Freon 11) & $75-69-4$ & $\mathrm{~N} / \mathrm{A}$ & $\mathrm{N} / \mathrm{A}$ & 2,000 & 1,000 \\
\hline Ethanenitrile (acetonitrile) & $75-05-8$ & 30,000 & 6,000 & 500 & 250 \\
\hline Propanone (acetone) & $67-64-1$ & 25,000 & 5,000 & 2,500 & 1,250 \\
\hline Furan & $110-00-9$ & $\mathrm{~N} / \mathrm{A}$ & $\mathrm{N} / \mathrm{A}$ & $\mathrm{N} / \mathrm{A}$ & N/A \\
\hline n-Pentane & $109-66-0$ & 14,000 & 2,800 & 1,500 & 750 \\
\hline 2-Propanol & 67-63-0 & 20,000 & 4,000 & 2,000 & 1,000 \\
\hline Dichloromethane (methylene chloride) & $75-09-2$ & 130,000 & 26,000 & 2,300 & 1,150 \\
\hline 1-Propanol & $71-23-8$ & 22,000 & 4,400 & 800 & 400 \\
\hline 2-Methyl pentane & $107-83-5$ & $\mathrm{~N} / \mathrm{A}$ & $\mathrm{N} / \mathrm{A}$ & $\mathrm{N} / \mathrm{A}$ & $\mathrm{N} / \mathrm{A}$ \\
\hline Propanenitrile & $107-12-0$ & 31,000 & 6,200 & $\mathrm{~N} / \mathrm{A}$ & N/A \\
\hline Butanal & $123-72-8$ & 14,000 & 2,800 & 1,400 & 700 \\
\hline 1-Hexene & $592-41-6$ & $\mathrm{~N} / \mathrm{A}$ & N/A & N/A & $\mathrm{N} / \mathrm{A}$ \\
\hline 2-Butanone & 78-93-3 & 14,000 & 2,800 & 3,000 & 1,500 \\
\hline n-Hexane & $110-54-3$ & 12,000 & 2,400 & 1,100 & 550 \\
\hline Trichloromethane (chloroform) & 67-66-3 & $\mathrm{N} / \mathrm{A}$ & N/A & 500 & 250 \\
\hline Tetrahydrofuran & 109-99-9 & 20,000 & 4,000 & 2,000 & 1,000 \\
\hline 1-Butanol & $71-36-3$ & 14,000 & 2,800 & 1,400 & 700 \\
\hline Benzene & $71-43-2$ & 12,000 & 2,400 & 500 & 250 \\
\hline $\begin{array}{l}\text { Tetrachloromethane (carbon } \\
\text { tetrachloride) }\end{array}$ & $56-23-5$ & $\mathrm{~N} / \mathrm{A}$ & N/A & 200 & 100 \\
\hline 1-Butanenitrile & 109-74-0 & 16,500 & 3,300 & $\mathrm{~N} / \mathrm{A}$ & N/A \\
\hline 3-Methyl hexane & $589-34-4$ & $\mathrm{~N} / \mathrm{A}$ & N/A & N/A & $\mathrm{N} / \mathrm{A}$ \\
\hline 2-Pentanone & $107-87-9$ & 15,000 & 3,000 & 1,500 & 750 \\
\hline n-Heptane & $142-82-5$ & 10,500 & 2,100 & 750 & 375 \\
\hline 1,4-Dioxane & 123-91-1 & 20,000 & 4,000 & 500 & 250 \\
\hline 4-Methyl-2-pentanone (hexone) & $108-10-1$ & 12,000 & 2,400 & 500 & 250 \\
\hline
\end{tabular}


HNF-3964, Rev. 2

Table 3-3. Required Analytes: Vapor Program Target Analytes (2 Sheets)

\begin{tabular}{|l|r|r|r|r|r|}
\hline \multicolumn{1}{|c|}{ Analyte } & $\begin{array}{r}\text { CAS } \\
\text { Number }\end{array}$ & $\begin{array}{r}\text { LFL } \\
\text { (ppmv) }\end{array}$ & $\begin{array}{r}\mathbf{2 0 \%} \\
\text { LFL } \\
\text { (ppmv) }\end{array}$ & $\begin{array}{r}\text { IDLH } \\
\text { (ppmv) }\end{array}$ & $\begin{array}{r}\mathbf{5 0 \%} \\
\text { IDLH } \\
\text { (ppmv) }\end{array}$ \\
\hline Toluene & $108-88-3$ & 11,000 & 2,200 & 500 & 250 \\
\hline 2-Hexanone & $591-78-6$ & N/A & N/A & 150 & 75 \\
\hline n-Octane & $111-65-9$ & 9,500 & 1,900 & 1,000 & 500 \\
\hline Tetrachloroethylene & $127-18-4$ & N/A & N/A & 150 & 75 \\
\hline Chlorobenzene & $108-90-7$ & 13,000 & 2,600 & 1,000 & 500 \\
\hline Ethylbenzene & $100-41-4$ & 8,000 & 1,600 & 800 & 400 \\
\hline m, p-Xylene & $106-42-3$ & 11,000 & 2,200 & 900 & 450 \\
\hline 3-Heptanone & $106-35-4$ & N/A & N/A & 1,000 & 500 \\
\hline 2-Heptanone & $110-43-0$ & 11,000 & 2,200 & 800 & 400 \\
\hline Cyclohexanone & $108-94-1$ & 11,000 & 2,200 & 700 & 350 \\
\hline Styrene & $100-42-5$ & 9,000 & 1,800 & 700 & 350 \\
\hline n-Nonane & $111-84-2$ & 8,500 & 1,700 & N/A & N/A \\
\hline o-Xylene & $95-47-6$ & 9,000 & 1,800 & 900 & 450 \\
\hline 1,1,2,2-Tetrachloroethane & $79-34-5$ & N/A & N/A & 100 & 50 \\
\hline 2-Octanone & $111-13-7$ & N/A & N/A & N/A & N/A \\
\hline n-Decane & $124-18-5$ & 7,500 & 1,500 & N/A & N/A \\
\hline 1,2,4-Trimethylbenzene & $95-63-6$ & 9,000 & 1,800 & N/A & N/A \\
\hline
\end{tabular}

Note:

$\mathrm{N} / \mathrm{A}=$ not available or not applicable 


\subsection{QUALITY ASSURANCE AND QUALITY CONTROL}

Vapor sampling and analysis shall be performed in accordance with approved quality assurance (QA) plans. These plans are required to meet the Hanford Analytical Services Quality Assurance Requirements Document (HASQARD) (DOE 1997) requirements. Validation of this compliance shall be verified either by a HASQARD assessment stating their quality program satisfactorily meets the appropriate requirements, or the quality program plan and applicable procedures will be submitted and approved prior to work performance on sampling or analytical work. SAS is currently working under the document Quality Assurance Management Plan Special Analytical Support, HNF-SD-WM-QAPP-034, ECN 618830 (Dormant 1998). Quality requirements for conducting Characterization Project sampling and analysis are described in Tank Waste Remediation System Characterization Project, Quality Policies (Board 1998) and this sampling and analysis plan. Characterization Project sampling and analysis shall be conducted in conformance with these QA requirements.

Processes, services, activities, and conditions adverse to quality which do not conform to requirements specified in this sampling and analysis plan or references herein shall be controlled to prevent inadvertent use. Nonconforming sampling and analysis processes shall be identified, controlled, reported, and dispositioned as required by PHMC (1998).

\subsection{LABORATORY OPERATIONS}

Analytical procedures that are to be used for this sample and analysis plan shall be written and approved prior to the performance of analytical work within the laboratory. Analytical quality control (QC) requirements are identified in Table 3-1. The laboratory shall also use calibration and calibration check standards appropriate for the analytical instrumentation being used (see DOE [1997] for definitions of QC samples and standards). The criteria presented are goals for demonstrating reliable method performance. It is understood that the laboratory will follow its internal QC system for required actions whenever QC failures occur. If sample QC failures occur or if all analyses cannot be performed (e.g., insufficient sample), analysts shall consult with supervisors/customers to determine the proper action. The laboratory should provide a suggested course of action at that time. All sample QC failures and limitations on the associated data shall be discussed in the narrative of the data report. Proper notification of all data not meeting QC requirements shall be included with the data.

\subsection{SAMPLE COLLECTION}

Sampling shall be performed in accordance with approved procedures and work plans included within the job control system. All data sheets and log entries completed during the performance of sampling shall be copied and included within the job control system package. 
HNF-3964, Rev. 2

Each sample identification number shall have the following format:

\section{VLXXX-YYY-ZZZZ}

where,

$\mathbf{V}$ indicates a vapor sample,

$\mathbf{L}=$ a letter code identifying the organization that prepared the sample container/sample media

$$
\begin{aligned}
& \mathrm{C}=\mathrm{CPO} \\
& \mathrm{P}=\mathrm{PNNL} \\
& \mathrm{S}=\mathrm{SAS},
\end{aligned}
$$

$\mathbf{X X X}=$ a three-digit code identifying the sample location

$$
\begin{aligned}
& 101=\operatorname{tank} 241-\mathrm{AZ}-101 \text { headspace } \\
& 102=\operatorname{tank} 241-\mathrm{AZ}-102 \text { headspace }
\end{aligned}
$$

YYY $=$ a three-digit sample code found in Tables 2-2 and 2-3

$\mathbf{Z Z Z Z}=$ a special laboratory-assigned code.

\subsection{SAMPLE CUSTODY}

Chain-of-custody will be carefully maintained to assure sample control at all times. 


\subsection{EXCEPTIONS, CLARIFICATIONS, AND ASSUMPTIONS}

\section{Equipment Blanks and Field Blanks}

Field Blanks are sampling devices prepared and handled in the same manner as the samples, but instead of tank gases, ambient air will be drawn into the canister. Equipment Blanks are sampling devices prepared and handled in the same manner as the samples, but instead of tank gases, ambient air will be drawn into the canister through the sampling equipment (tubing). 
HNF-3964, Rev. 2

\subsection{ORGANIZATION}

The organization and responsibility of key personnel involved with these tank characterization projects are listed in Table 6-1.

Table 6-1. Project Key Personnel

\begin{tabular}{|c|c|c|}
\hline Responsibility & Organization & Individual \\
\hline $\begin{array}{l}\text { Data Assessment and } \\
\text { Interpretation manager }\end{array}$ & $\begin{array}{l}\text { TWRS Process Engineering } \\
\text { (LMHC) }\end{array}$ & J. G. Field, 376-3753 \\
\hline $\begin{array}{l}\text { Process Engineering point of } \\
\text { contact for vapor sampling }\end{array}$ & $\begin{array}{l}\text { TWRS Process Engineering } \\
\text { (LMHC) }\end{array}$ & A. M. Templeton, 373-5589 \\
\hline Operations point of contact ${ }^{1}$ & Operations Program (LMHC) & G. P. Hopkins, 373-1166 \\
\hline Cognizant Engineer & $\begin{array}{l}\text { Double Shell Tank } \\
\text { Engineering (LMHC) }\end{array}$ & G. R. Tardiff, 373-5539 \\
\hline $\begin{array}{l}\text { Vapor sampling cognizant } \\
\text { engineer }\end{array}$ & $\begin{array}{l}\text { Characterization Field } \\
\text { Engineering (LMHC) }\end{array}$ & D. D. Wanner, 373-3297 \\
\hline $\begin{array}{l}\text { SAS vapor sampling and } \\
\text { analysis project manager }\end{array}$ & $\begin{array}{l}\text { Special Analytical Support } \\
\text { (NHC) }\end{array}$ & L. L. Lockrem, 373-4771 \\
\hline $\begin{array}{l}\text { SAS vapor sampling and } \\
\text { analysis technical contact }\end{array}$ & $\begin{array}{l}\text { Special Analytical Support } \\
\text { (COGEMA) }\end{array}$ & R. S. Viswanath, 376-9223 \\
\hline $\begin{array}{l}\text { PNNL vapor sample analysis } \\
\text { project manager }\end{array}$ & PNNL & K. L. Silvers, 372-4828 \\
\hline $\begin{array}{l}\text { PNNL vapor sample analysis } \\
\text { technical contact }\end{array}$ & PNNL & $\begin{array}{l}\text { J. C. Evans, 376-0934, } \\
\text { J. L. Huckaby, 376-5524 }\end{array}$ \\
\hline $\begin{array}{l}\text { Industrial Hygiene and Safety } \\
\text { point of contact }\end{array}$ & $\begin{array}{l}\text { TFFO Field Safety Services } \\
(\mathrm{LMHC})\end{array}$ & $\begin{array}{l}\text { K. M. Bowen, 372-3667 } \\
\text { N. K. Butler, 376-5795 }\end{array}$ \\
\hline $\begin{array}{l}\text { Double-Shell Tank Farm } \\
\text { point of contact }\end{array}$ & Tank Farm Operations & $\begin{array}{l}\text { Double-Shell Tank Farm } \\
\text { Operations shift manager, } \\
373-2689\end{array}$ \\
\hline $\begin{array}{l}\text { Waste Feed Delivery point of } \\
\text { contact }\end{array}$ & Tank Waste Retrieval & R. D. Potter, 373-9315 \\
\hline $\begin{array}{l}\text { Environmental point of } \\
\text { contact }\end{array}$ & $\begin{array}{l}\text { Environmental } \\
\text { Permits/Policy (LMHC) }\end{array}$ & G. M. Crummel, 373-5175 \\
\hline
\end{tabular}

Notes:

'Test Director will be appointed by the complex facility manager. 


\section{HNF-3964, Rev. 2}

\subsection{DELIVERABLES}

Sampling and analytical results shall be reported as Format VI reports. Any analyte exceeding the notification limit prescribed in Table 3-1 shall also be reported as a Format I report. In addition, SAS shall provide Format II reports to the analytical laboratories as described in Section 7.2.

\subsection{FORMAT I REPORTING}

Table 3-1 contains the notification limits for specific analytes. Analytes that exceed notification limits shall be reported by the Project Manager or delegate by calling the Double-Shell Tank Farms Operations shift manager as soon as the data are obtained and reviewed by the responsible scientist. This verbal notification must be followed within one hour by electronic notification to the Double-Shell Tank Farms Operations shift manager, the Industrial Hygiene and Safety point of contact, the TWRS Process Engineering Data Assessment and Interpretation manager, the airlift circulator operations point of contact and the Process Engineering point of contact for vapor sampling. A further review of the data, including quality control results and additional analyses for verification purposes may be contracted with the performing laboratory by either a revision to this sampling and analysis plan or by a letter.

\subsection{FORMAT II REPORTING}

Information on the sampling shall be reported by the CPO sampling team and WSCF as Format II reports to the SAS and PNNL laboratories and the point of contact in Process Engineering. CPO will provide the sample collection sequence and volumes, start and stop times for the collection of each sample, the TOC reading at the start of the collection of each sample, verification of equipment and field blank use, any anomalous sampling conditions, and WSCF will provide the results of the tritium trap and particulate filter analysis. This report is to accompany, if possible, the shipment of samples. Alternatively, this sampling report may be delivered to SAS and PNNL within 48 hours after the samples have been relinquished from the samplers.

\subsection{FORMAT VI REPORTING}

The Format VI report shall consist of two deliverables, preliminary analytical results and a final data package. Report Chemical Abstract Service (CAS) numbers in mg/L and ppb.

Preliminary sampling and analytical data shall be delivered within two weeks of the receipt of the samples at the laboratory. The preliminary data shall consist of, at a minimum, data tables reporting sample collection data, tritium trap and particulate filter analysis results, and the results of each analysis performed by the analytical laboratory (SAS or PNNL). The following individuals shall be on distribution for the preliminary results: G. M. Crummel, C. H. Mulkey, R. D. Potter, A. M. Templeton, J. Jo, and J. H. Rasmussen. 
HNF-3964, Rev. 2

A data package shall be issued as a supporting document within ten weeks of the receipt of the samples at the laboratory. The data package shall contain the elements listed in Table 7-1. The following individuals shall be on distribution for the entire data package: R. A. Bechtold, G. M. Crummel, R. D. Potter, A. M. Templeton, J. H. Rasmussen, and C. A. Simonen.

Table 7-1. Data Package Required Elements ${ }^{1}$ (2 Sheets)

\begin{tabular}{|l|}
\hline \multicolumn{1}{|c|}{ Prefatory Elements } \\
\hline Executive summary \\
\hline Table of contents \\
\hline List of abbreviations and acronyms \\
\hline Quality assurance data package review results \\
\hline Non-conformance reports \\
\hline \\
\hline Sampling case narrative \\
\hline Sample summary and event chronology \\
\hline Sampling procedures table \\
\hline Sampling logbook table \\
\hline Field data \\
\hline Radiation screening results \\
\hline Chain of custody forms \\
\hline Trip blank summary \\
\hline Analys spectrometer instrument tune report \\
\hline Analytical procedures table \\
\hline Data qualifier flag translation table \\
\hline Target analytes concentration table \\
\hline Tentatively identified compound concentration table \\
\hline
\end{tabular}


HNF-3964, Rev. 2

Table 7-1. Data Package Required Elements ${ }^{1}$ (2 Sheets)

\begin{tabular}{|l|}
\hline Target analyte initial calibration table \\
\hline Internal standards area counts table \\
\hline Laboratory control sample results table \\
\hline Surrogate compounds results table \\
\hline Quantitation reports \\
\hline Chromatograms \\
\hline Mass spectra of reported tentatively identified compounds \\
\hline Note: \\
'Include all elements as applicable.
\end{tabular}

In addition to the data package, an electronic version of the analytical results shall be provided to the Tank Vapor Database representative within 4 calendar days from the day that the final data package is issued. The data must be available to the Washington State Department of Ecology within 7 calendar days of release of the data package. The electronic version shall be in the standard electronic format (Bobrowski and Simonen, 1999). 
HNF-3964, Rev. 2

\subsection{CHANGE CONTROL}

Under certain circumstances, it may become necessary for the performing laboratory to make decisions concerning a sample without review of the data by the customer or the Characterization Project. All significant changes shall be documented by TWRS Process Engineering via an engineering change notice to this SAP or by a letter. All changes shall also be clearly documented in the final data report. Insignificant changes may be made by placing a notation in the permanent record (i.e., note change in log book or memo to file). Significance is determined by the Process Engineering point of contact for vapor sampling.

At the request of the Characterization Project, additional analysis of sample material from this characterization project shall be performed following a revision of this SAP or issuance of a letter. 


\section{HNF-3964, Rev. 2}

\subsection{REFERENCES}

Board, D. C., 1998, Tank Waste Remediation System, Characterization Project, Quality Policies, HNF-SD-WM-QAPP-025, Rev. 4, Lockheed Martin Hanford Corp. for Fluor Daniel Hanford, Inc., Richland, Washington.

Bobrowski, S .F., and C. A. Simonen, 1999, Standard Electronic Format Specification for Tank Vapor Data MSEXCEL Spreadsheets: Version 1.0, HNF-3815, Rev. 0, prepared by Pacific Northwest National Laboratory for Lockheed Martin Hanford Corporation, Richland, Washington

DOE, 1997, Hanford Analytical Services Quality Assurance Requirements Document, DOE/RL-96-68, Rev. 1, U.S. Department of Energy, Richland Field Office, Richland, Washington.

Dormant, C., 1998, Quality Assurance Management Plan Special Analytical Support, HNF-PRO-298, Rev. 1, Fluor Daniel Hanford, Inc., Richland, Washington.

LMHC, 1999a, AZ-101 and AZ-102 Airlift Circulator Operation Instructions, Process Memo \#2E98-082, East Tank Farm Engineering, January 26, 1998, Lockheed Martin Hanford Corporation, Richland, Washington..

LMHC, 1999b, Gas Sampling For AZ-101 and AZ-102 Airlift Circulator Operation, Process Memo \#2E99-001, East Tank Farm Engineering, January 26, 1998, Lockheed Martin Hanford Corporation, Richland, Washington.

Mulkey, C. H., and K. D. Markillie, 1995, Data Quality Objectives for Regulatory Requirements for Hazardous and Radioactive Air Emissions Sampling and Analysis, WHC-SD-WM-DQO-021, Rev. 0, Westinghouse Hanford Company, Richland, Washington.

PHMC, 1998, Nonconforming Item Reporting and Control, HNF-PRO-298, Rev. 1, Fluor Daniel Hanford, Inc., Richland, Washington. 